\title{
Aperfeiçoamento da Técnica de Soldagem Pontual por Friç̧ão (FSpW) para União de Poliamida 6 e Laminado de Poliamida 66 com Fibra de Carbono
}

\author{
Improvement of the Friction Spot Welding (FSpW) to join Polyamide 6 and Polyamide 66/Carbon Fiber Laminate
}

\author{
Joel Gonçalves ${ }^{1}$, Jorge Fernandez dos Santos ${ }^{2}$, Leonardo Bresciani Canto ${ }^{3}$, Sergio de Traglia Amancio Filho ${ }^{2,4}$ \\ ${ }^{1}$ Universidade Federal de São Carlos (UFSCar), Programa de Pós-Graduação em Ciência e Engenharia de Materiais (PPG-CEM), \\ São Carlos, São Paulo, Brasil, joel13br@hotmail.com \\ ${ }^{2}$ Helmholtz-Zentrum Geesthacht (HZG), Institute of Materials Research/Materials Mechanics/Solid State Joining Processes, \\ Geesthacht, Schleswig-Holstein, Germany \\ ${ }^{3}$ Universidade Federal de São Carlos (UFSCar), Departamento de Engenharia de Materiais (DEMa), São Carlos, São Paulo, Brasil \\ ${ }^{4}$ Helmholtz-Zentrum Geesthacht (HZG), Institute of Materials Research/Materials Mechanics/Advanced Polymer-Metal Hybrid \\ Structures Geesthacht, Schleswing-Holstein, Germany,sergio.amancio@hzg.de
}

\begin{abstract}
Resumo
A Soldagem Pontual por Fricção ('Friction Spot Welding - FSpW') é uma técnica inovadora que foi desenvolvida e patenteada em 2005 pelo centro de pesquisa alemão Helmholtz Zentrum Geesthacht (HZG). A técnica apresenta ciclos rápidos, baixo custo operacional e gera soldas com alto desempenho mecânico. Foi inicialmente projetada para a soldagem de ligas metálicas leves. A soldagem de polímeros é alvo de pesquisas recentes e a soldagem de compósitos poliméricos por FSpW ainda é inédita. Este estudo demonstrou a viabilidade técnica da soldagem de chapa de poliamida 6 (PA6) e laminado de poliamida 66 e fibra de carbono (CF-PA66) por FSpW. O histórico térmico, o acabamento superficial, a microestrutura da região da solda e a resistência mecânica ao cisalhamento de juntas sobrepostas PA6/CF-PA66 foram investigados. O aumento do tamanho do anel de fixação da ferramenta original, projetada para a soldagem de metais, possibilitou a seleção de parâmetros de soldagem que resultaram em grande aporte térmico, resultando no aumento da área soldada, num menor entalhe (inscrição) deixado pela ferramenta na amostra, produzindo melhoria no acabamento superficial da solda. Essa otimização resultou em uma junta PA6/CF-PA66 com resistência ao cisalhamento de 35 MPa (2196 N), com fratura predominante na placa superior de PA6.
\end{abstract}

Palavras-chave: Friction Spot Welding; FSpW; Soldagem; Poliamida; Laminado de poliamida e fibra de carbono, Compósito.

Abstract: Friction Spot Welding is an innovative technique developed and patented in 2005 by the research center Helmholtz Zentrum Geesthacht (HZG). Advantages of the process include fast joining cycles, low operational costs and good mechanical performance. It was originally developed to weld lightweight metals and has been continuously improved to weld polymers. There are only few studies on friction spot welding of thermoplastics and the welding of polymeric composites by FSpW is still unpublished. This study demonstrated the technical feasibility of welding a polyamide 6 (PA6) plate over a carbon fiber polyamide 66 laminate (CF-PA66) by FSpW. The thermal history, the surface finish, the microstructure of the weld region and the mechanical shear strength of PA6/CF-PA66 lap joints were investigated. The increase of the diameter of the clamping ring - originally designed for welding lightweight metals - led to the selection of welding parameters that result in high heat input, resulting in an increased welded area, and lower notch left by the tool in the sample that improved the surface finish of the weld. This optimization resulted in a PA6/CF-PA66 joint with lap shear strength of $35 \mathrm{MPa}(2196 \mathrm{~N})$, with fracture occurring mainly in the top plate of PA6.

Key-words: Friction Spot Welding; FSpW; Polyamide; Polyamide 6.6/carbon fiber laminate, Composites.

\section{Introdução}

O emprego crescente de polímeros e compósitos de engenharia nas indústrias automobilística, aeronáutica e de eletroeletrônicos tem como principal motivo as novas políticas

Recebido em 04/02/2014, texto final em 01/03/2014. de redução da emissão de gases na atmosfera que visam aumentar ao máximo a eficiência entre peso e desempenho dos materiais [1,2]. Processos de soldagem para esses materiais são indispensáveis nesse desenvolvimento, pois estruturas feitas em uma única peça são normalmente impraticáveis do ponto de vista técnico e econômico, devido às características restritivas como design complexo, tamanho, indisponibilidade de tecnologias de processamento e, principalmente, a necessidade da combinação de diferentes materiais na mesma peça. Isso 
motiva o desenvolvimento de técnicas rápidas, confiáveis e de alta produtividade para a união entre polímeros e compósitos.

A soldagem pontual por fricção ('Friction Spot Welding - FSpW') é uma técnica inovadora que foi desenvolvida e patenteada em 2005 pelo centro de pesquisa Helmholtz Zentrum Geesthacht (HZG), na Alemanha [3]. A FSpW foi inicialmente projetada para a soldagem de ligas metálicas leves como o alumínio [4] e magnésio [5] e, devido às suas vantagens singulares como ciclos rápidos, baixos custos operacionais e obtenção de soldas com bom desempenho mecânico, a FSpW tem sido estudada para a soldagem de outros materiais, similares ou dissimilares, envolvendo metais, polímeros e compósitos poliméricos [6,7].

AFSpW é uma evolução da técnica 'Friction Stir Spot Welding - FSSW'. A ferramenta da FSSW é composta por duas partes fixas e não consumíveis (pino e o rebaixo). As principais etapas do processo FSSW são ilustradas na Figura 1-a. Primeiramente, as partes a serem soldadas são fixadas sobre pressão. A ferramenta, em alta rotação, é inserida na área a ser soldada, o que gera aumento local da temperatura da amostra por fricção. No caso de metais, a temperatura máxima alcançada varia de 70 a $90 \%$ do ponto de fusão do material [8]. Para termoplásticos, onde pouca informação ainda está disponível na literatura, a temperatura atingida deve ultrapassar a temperatura de transição vítrea, no caso de polímeros amorfos, e a temperatura de fusão cristalina, para os polímeros semicristalinos [9]. No final do processo, a ferramenta é retraída e a solda se consolida, deixando um entalhe (impressão feita pelo pino) no centro da solda [10,11]. Bilici [12] e Arici e Mert [9] avaliaram a soldabilidade de juntas de polipropileno (PP) sobrepostas enquanto Bilici e Yükler [13] e Bilici, Yükler e Kurtulmuş [14] avaliaram a soldabilidade de polietileno de alta densidade (HDPE) utilizando a técnica FSSW. Porém, o entalhe deixado ao final do processo na amostra serve como um ponto concentrador de tensões que pode acarretar na formação de trinca, além de depreciar o acabamento superficial da solda.

A principal diferença da FSpW para a FSSW reside no desenho da ferramenta. A ferramenta da FSpW foi projetada para solucionar o problema do entalhe criado pela retração da ferramenta FSSW. Essa consiste em três diferentes partes com movimentos independentes (Figura 1-b). A parte externa é chamada anel de fixação ('clamping ring') e tem a tarefa de manter a pressão constante sobre as amostras. A parte interna é chamada pino ('pin') e a parte intermediária de camisa ('sleeve') e ambas possuem a tarefa de gerar aquecimento através da fricção. A técnica $\mathrm{FSpW}$ pode ser utilizada em duas variantes diferentes: Camisa-Penetrante ('Sleeve-Plunge') e PinoPenetrante ('Pin-Plunge'), que diferem basicamente pela parte penetrante da ferramenta [15]. A variante Camisa-Penetrante é a mais adotada por gerar áreas soldadas maiores e, portanto, soldas mais resistentes. Primeiramente as amostras são fixadas em sobreposição e pressionadas contra o anel de fixação enquanto o pino e a camisa começam a rotacionar em um mesmo sentido a determinada velocidade angular. A camisa penetra a amostra e a fricção gera aquecimento suficiente para amolecer o material na região da solda. Ao mesmo tempo em que a camisa penetra as amostras o pino retrai criando um espaço para o preenchimento do material amolecido. Após o limite de penetração ser atingido, a camisa e o pino retornam para sua posição original e o material amolecido é realocado no espaço deixado pela ferramenta. No final do ciclo de soldagem a ferramenta é mantida em contato com as amostras e a solda é resfriada sobre pressão evitando contração diferencial durante sua consolidação.

A primeira tentativa do uso da FSpW para a soldagem de termoplásticos foi realizada por Oliveira et al. [16,17] em poli(metacrilato de metila) (PMMA). As soldas obtidas apresentaram resistência ao cisalhamento comparáveis e muitas vezes superiores às obtidas por ultrassom, por soldagem térmica e por soldagem por microondas. A união de termoplásticos pela técnica FSpW ainda é alvo de pesquisas recentes, com poucos trabalhos publicados, e o seu uso para a soldagem de compósitos termoplásticos é inédita.

As dimensões das ferramentas usuais da FSpW foram obtidas da experiência prévia com soldas metálicas e apresentam algumas limitações para emprego em polímeros e compósitos poliméricos. $\mathrm{O}$ anel de fixação tradicional tem um diâmetro externo pequeno (14,5 mm), que acaba por ser inserido na superfície da amostra polimérica quando soldada, devido ao seu menor módulo de elasticidade com relação aos materiais metálicos. Esse efeito cria um entalhe geométrico na superfície da solda cuja profundidade é proporcional a energia térmica gerada. Observa-se com isso a perda de polímero fundido, devido sua baixa viscosidade, para fora do anel de fixação e para o interior da ferramenta. Desse modo, o acabamento superficial da solda é depreciado e a quantidade de material na região soldada diminui levando à redução da resistência mecânica da junta. Além disso, há o fato de que o material perdido para o interior do equipamento obstrui o sistema de resfriamento levando a uma limitação do número de soldas, desgaste acelerado da superfície da ferramenta e a um maior consumo de energia devido ao aumento do torque necessário para sua rotação. Outro diferencial na soldagem de polímeros por $\mathrm{FSpW}$, em relação aos metais, está relacionado à sua reduzida condutividade térmica necessitando, portanto, de maior energia térmica e/ou tempos mais longos de soldagem para a geração de soldas sem defeitos volumétricos e com áreas soldadas maiores. Sendo assim, é esperado que o uso de anéis de fixação com maiores diâmetros venha a flexibilizar o intervalo de soldagem, viabilizando o uso de maiores aportes térmicos.

Este estudo demonstrou a viabilidade do emprego da FSpW para a união de chapa de poliamida 6 (PA6) e laminado de poliamida 66 com fibra de carbono (CF-PA66) por meio da otimização de parâmetros de processo e modificação do tamanho do anel de fixação da ferramenta original. Foram investigados o histórico térmico das soldas produzidas, o acabamento superficial, a microestrutura da região da solda e a resistência mecânica ao cisalhamento de uniões PA6/CF-PA66 sobrepostas. Observou-se que a modificação na ferramenta diminuiu o entalhe ao final do processo e permitiu a escolha de parâmetros que aumentam o aporte térmico durante a soldagem, garantindo assim, menor efeito concentrador de tensão e maior resistência mecânica devido à geração de áreas soldadas maiores. 

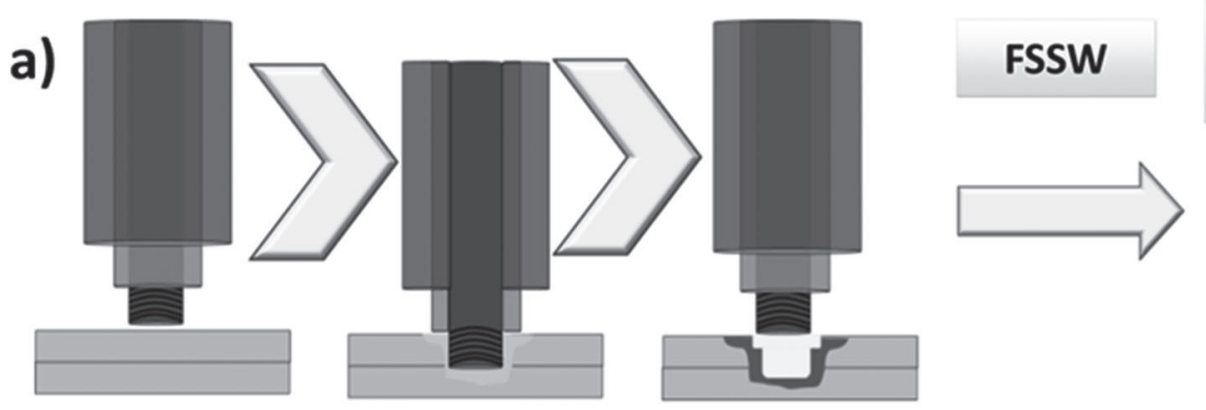

\section{Entalhe formado pela ferramenta}
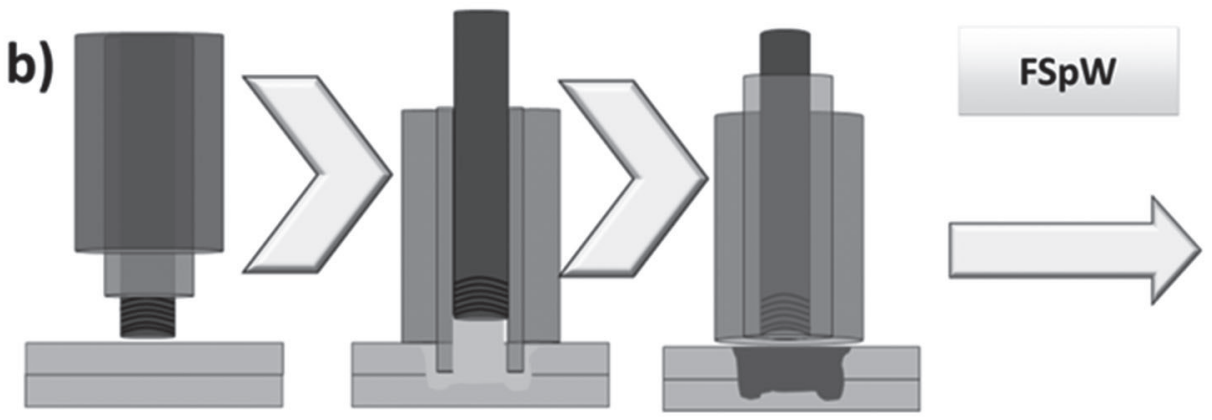

\section{Ausência de entalhe}

Figura 1. Esquema ilustrativo da soldagem por FSSW (a) e FSpW (b)

Tabela 1. Propriedades da chapa extrudada de PA6 marca Erlaton 6 da empresa Arthur Krüger [18]

\begin{tabular}{|c|c|c|c|}
\hline Propriedades & Valores & Unidades & Norma \\
\hline Temperatura de transição vítrea - $T_{g}$ & 60 & ${ }^{\circ} \mathrm{C}$ & - \\
\hline Temperatura de cristalização - $T_{c}$ & 180 & ${ }^{\circ} \mathrm{C}$ & \\
\hline Temperatura de fusão $-T_{m}$ & 220 & ${ }^{\circ} \mathrm{C}$ & \\
\hline Resistência à tração & 76 & $\mathrm{MPa}$ & ISO 527 \\
\hline Módulo & 3.250 & $\mathrm{MPa}$ & ISO 527 \\
\hline
\end{tabular}

Tabela 2. Propriedades do laminado de poliamida 66 com fibras de carbono (CF-PA66) marca TEPEX Dynalite 201 da empresa BOND Laminates [19]

\begin{tabular}{l|c|c|c|}
\hline \multicolumn{1}{|c|}{ Propriedades } & Valores & Unidades & Norma \\
\hline Densidade & 1,43 & $\mathrm{~g} / \mathrm{cm}^{3}$ & ${ }^{\circ} \mathrm{C}$ \\
\hline Temperatura de transição vítrea $-\mathbf{T}_{\mathbf{g}}$ & 70 & ${ }^{\circ} \mathrm{C}$ \\
\hline Temperatura de fusão $-\mathbf{T}_{\mathbf{m}}$ & 260 & $\mathrm{MPa}$ & - \\
\hline Resistência à tração Longitudinal & 785 & $\mathrm{MPa}$ & $\mathrm{ISO} 527-4 / 5$ \\
\hline Resistência à tração Transversal & 725 & $\mathrm{MPO} 527-4 / 5$ \\
\hline Módulo & 53.000 & $\mathrm{MPa}$ & ISO 527-4/5 \\
\hline
\end{tabular}




\section{Materiais e Métodos}

Os materiais utilizados nesta pesquisa são baseados em poliamidas.

Utilizou-se chapa extrudada de poliamida 6 (PA6) com $4 \mathrm{~mm}$ de espessura da marca Erlaton 6 produzida pela empresa Arthur Krüger, cujas propriedades são apresentadas na Tabela 1.

O compósito utilizado é um laminado de 2,0 mm de espessura de poliamida 66 reforçada por um tecido de fibras de carbono, produzido pela empresa alemã Bond Laminates. O grade do compósito é chamado TEPEX possuindo $49 \%$ em peso de fibra de carbono distribuídas em 9 camadas, sendo duas camadas do grade (201_C200) e sete camadas do grade (201_C190) na configuração $\overline{2} / 2$ twill. Suas propriedades são apresentadas na Tabela 2 .

As amostras foram cortadas a partir da chapa de PA6 e do laminado CF-PA66 nas dimensões de 25,4 mm de largura e 100 mm de comprimento, baseadas na norma ASTM D3163-01 [20].

Foram produzidas soldas por FSpW na configuração CamisaPenetrante utilizando um equipamento em escala de laboratório (RPS 100, Harms \& Wende) no centro de pesquisa Helmholtz Zentrum Geesthacht (HZG) na Alemanha. As amostras foram sobrepostas com a chapa de PA6 sobre o laminado de CFPA66 e fixadas por um porta-amostra que garantiu o contato íntimo entre suas superfícies. Duas condições experimentais de soldagens (Tabela 3) foram selecionadas baseadas num intervalo de parâmetros de soldagem otimizado. A condição A utilizou a ferramenta tradicional que dispunha de um anel de fixação com 14,5 mm de diâmetro externo. A condição B utilizou uma nova ferramenta com anel de fixação de $22 \mathrm{~mm}$ de diâmetro externo. A profundidade de penetração da ferramenta para ambas as condições (A e B) foi ajustada em 3,8 mm de modo que a ferramenta não ultrapassasse a chapa superior de PA6 com $4 \mathrm{~mm}$ de espessura. A velocidade de rotação da ferramenta para ambas as condições foi ajustada em $1500 \mathrm{rpm}$ e o tempo de consolidação em $20 \mathrm{~s}$. Com o objetivo de avaliar a influência da nova dimensão de ferramenta sobre a intervalo de parâmetros de soldagem, o tempo de permanência da ferramenta sob máxima penetração assim como a pressão da condição $\mathrm{B}$ foram estabelecidas intencionalmente maiores que os valores da condição A. Ao final do processo de soldagem, as amostras foram mantidas fixadas ao porta-amostra por três minutos, tempo este suficiente para que a junta atingisse a temperatura ambiente.

Medidas de temperatura processual foram feitas utilizando uma câmera de infravermelho (ImageIR 8300, Infratec $\mathrm{GmbH}$ ) calibrada na faixa de temperatura entre $20-300^{\circ} \mathrm{C}$. A temperatura analisada nesse trabalho corresponde à máxima temperatura superficial do ponto de solda, coletada a partir do momento em que a ferramenta se afasta da amostra, desconsiderando o tempo de consolidação.

A microestrutura de secções transversais extraídas do centro das soldas foi analisada em um microscópio ótico Leica Q550IW, acoplado a um computador com software QWIN de visualização fotográfica. Para a preparação das amostras realizou-se um corte seccional na região soldada e em seguida, as amostras foram embutidas em resina epoxi de cura a frio (Buehler Epoxicure). As etapas de lixamento e polimento foram executados em uma politriz automática Buehler Phoenix 4000 para posterior visualização no microscópio.

A resistência mecânica das juntas soldadas foi avaliada por ensaios de cisalhamento de juntas sobrepostas ('single lap shear'). O teste foi realizado em uma máquina universal de ensaios Zwick/Roel 1478, de acordo com a norma ASTM D316301 , com velocidade de travessa de $1,27 \mathrm{~mm} / \mathrm{min}$ em temperatura ambiente. Uma área nominal circular de $63,6 \mathrm{~mm}^{2}$, calculada a partir do diâmetro externo da camisa, foi considerada para os cálculos da tensão de cisalhamento das juntas sobrepostas.

\section{Resultados e Discussão}

As temperaturas máximas na região da solda das juntas PA6/ CF-PA66 produzidas por FSpW nas condições A e B e a área utilizada para o monitoramento da temperatura são mostradas na Figura 2. A superfície da região da solda alcançou temperaturas de $280^{\circ} \mathrm{C}$ e $352^{\circ} \mathrm{C}$ para as condições $\mathrm{A}$ e $\mathrm{B}$, respectivamente. Essas temperaturas ultrapassam as temperaturas de fusão $\left(\mathrm{T}_{\mathrm{m}}\right)$ das poliamidas (Tabelas 1 e 2). Acredita-se que a temperatura interna da região de solda, cujo monitoramento experimental não é possível devido à dinâmica do processo, seja ainda maior que a medida por infravermelho, garantindo assim a plastificação completa dos polímeros durante a soldagem. A condição B produziu maior temperatura na região da solda $\left(352^{\circ} \mathrm{C}\right) \mathrm{em}$ relação à condição $\mathrm{A}\left(280^{\circ} \mathrm{C}\right)$ que se deve à utilização de um maior tempo de soldagem (maior tempo sob cisalhamento) e também devido à maior força de fixação utilizada que gerou maior calor por fricção.

O acabamento superficial das soldas PA6/CF-PA66 foi utilizado como variável de resposta para a avaliação dos parâmetros de soldagem (condições A e B). As fotografias das soldas PA6/CF-PA66 produzidas por FSpW nas condições A e B são apresentadas na Figura 3. Pode-se observar que para a solda produzida na condição A (anel de fixação com menor diâmetro) tem-se uma solda com um entalhe (Figura 3-a) formado pela inscrição da ferramenta durante a soldagem. Esse efeito ocorre devido ao fato de que a relação entre a força exercida e a área do anel de fixação gera uma pressão demasiada grande sobre a

Tabela 3. Condições experimentais de soldagem das uniões PA6/CF-PA66 por FSpW

\begin{tabular}{|l|c|c|c|c|c|c|}
\hline & $\begin{array}{c}\text { Diâmetro do anel } \\
\text { de fixação } \\
(\mathrm{mm})\end{array}$ & $\begin{array}{c}\text { Velocidade de } \\
\text { rotação } \\
(\mathrm{rpm})\end{array}$ & $\begin{array}{c}\text { Profundidade } \\
\text { de penetração } \\
(\mathrm{mm})\end{array}$ & $\begin{array}{c}\text { Tempo sob } \\
\text { penetração máxima } \\
(\mathrm{s})\end{array}$ & $\begin{array}{c}\text { Força de } \\
\text { fixação } \\
(\mathrm{kN})\end{array}$ & $\begin{array}{c}\text { Tempo de } \\
\text { consolidação } \\
(\mathrm{s})\end{array}$ \\
\hline Condição A & 14,5 & 1500 & 3,8 & 2 & 8,5 & 20 \\
\hline Condição B & 22,0 & 1500 & 3,8 & 8 & 15 & 20 \\
\hline
\end{tabular}



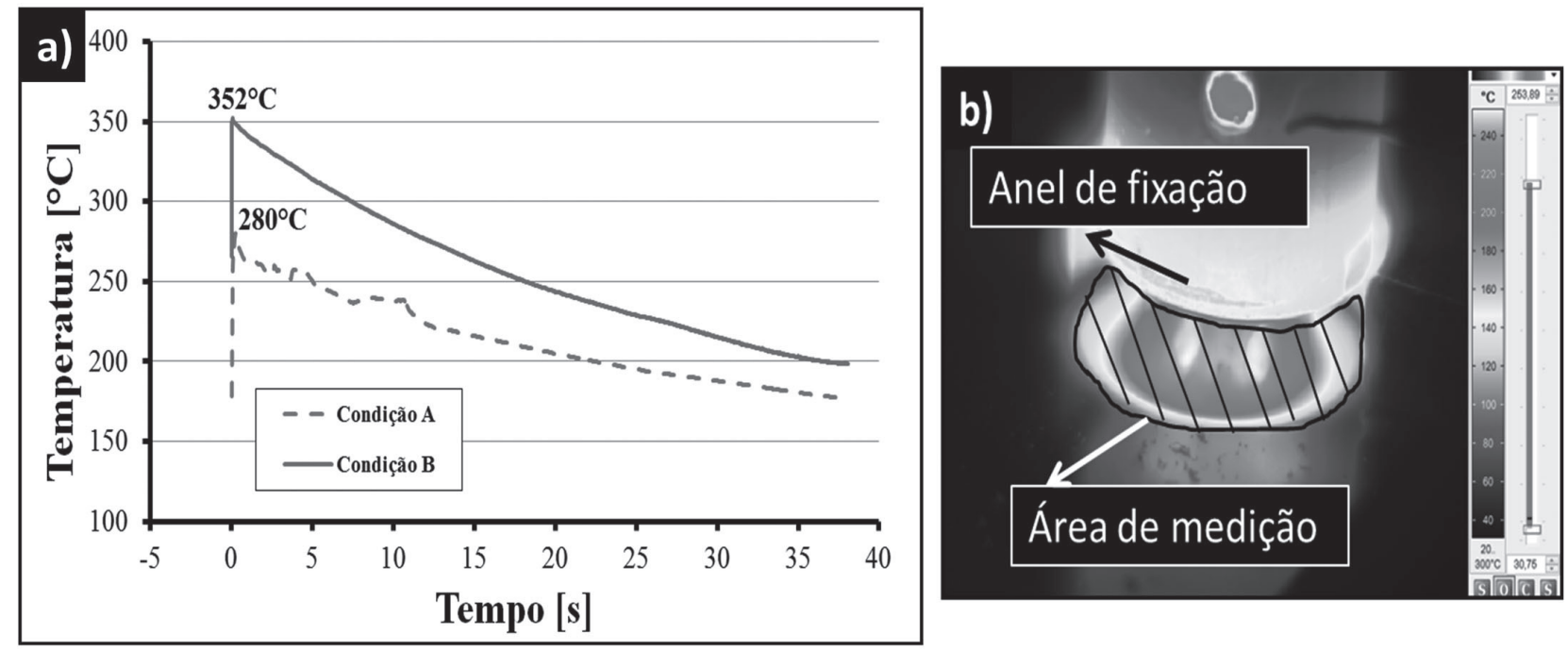

Figura 2. a) Temperaturas máximas na região da solda das juntas PA6/CF-PA66 produzidas por FSpW nas condições A e B; b) Área utilizada para o monitoramento da temperatura
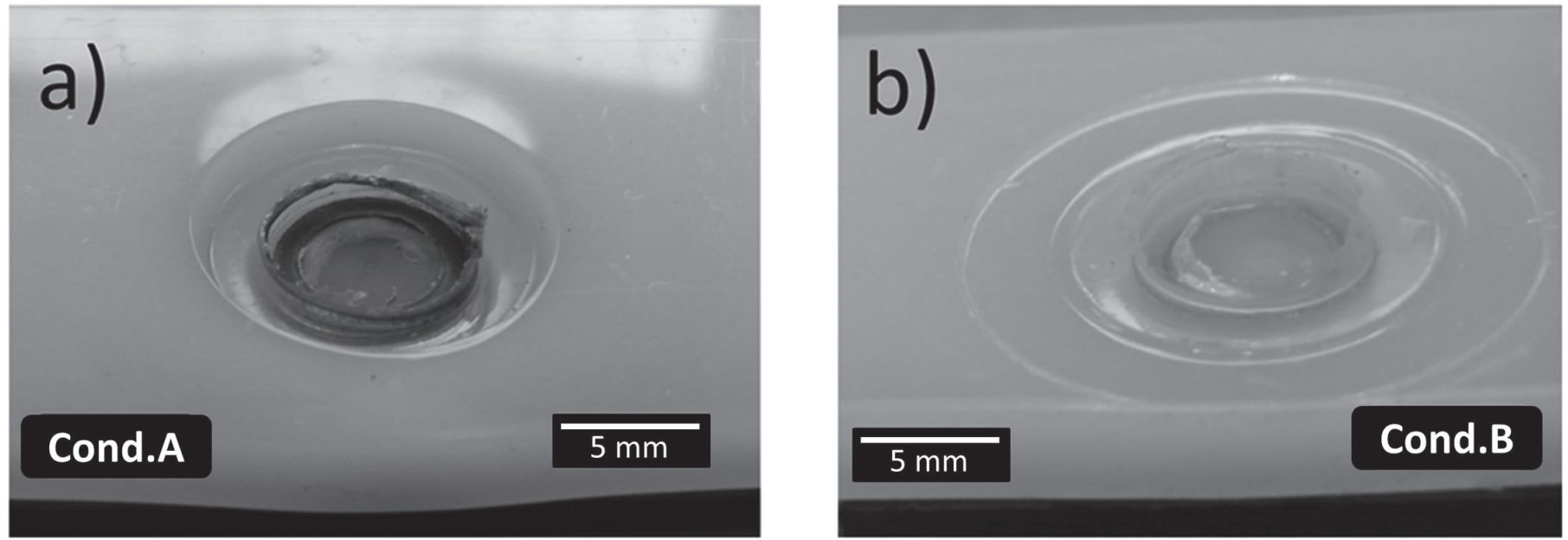

Figura 3. Acabamento superficial das soldas PA6/PA66-CF produzidas por FSpW na condição A utilizando uma ferramenta com diâmetro do anel de fixação de 14,5 mm (a) e na condição B utilizando uma ferramenta com diâmetro do anel de fixação de 22 mm (b)

amostra. O entalhe deixado pela ferramenta, além de depreciar o acabamento superficial, também pode atuar como um ponto concentrador de tensão (nucleação prematura de trinca) o que levaria à diminuição da resistência mecânica da solda. A solda produzida na condição B com uma ferramenta com anel de fixação com diâmetro maior (Figura 3-b) apresentou somente uma leve inscrição da divisão das partes da ferramenta na amostra. A ausência desse entalhe pode ser explicada pelo fato de que a força aplicada sobre a amostra é melhor distribuída pela nova área da ferramenta e pelo fato de a área sob a ferramenta estar mais distante do centro da amostra que se encontra a uma temperatura elevada.

A Figura 4 apresenta a microestrutura da secção de corte das juntas PA6/CF-PA66 produzidas por FSpW nas condições A (Figura 4-a) e B (Figura 4-b). A solda produzida na condição A (anel de fixação com diâmetro menor) apresentou entalhe com profundidade de $3,08 \mathrm{~mm}$, enquanto que a solda produzida na condição B (anel de fixação com diâmetro maior) apresentou entalhe menor, com profundidade de 0,74 $\mathrm{mm}$. Pode-se observar também que na condição A o entalhe ocasionou o aumento da penetração máxima (configurada pelo equipamento para ser de $3,8 \mathrm{~mm}$ ), chegando a atingir a chapa do laminado. Este entalhe além de diminuir a precisão do parâmetro de profundidade de penetração ocasiona maior desgaste da ferramenta devido à maior fricção entre a camisa e as fibras de carbono do laminado. Outro dado importante obtido das imagens da secção de corte é o volume de material que foi expelido da região de solda. Podese observar que a área da seção transversal do entalhe da solda produzida na condição A é de $34,2 \mathrm{~mm}^{2}$ enquanto que na condição $\mathrm{B}$ essa área do entalhe é de $8,6 \mathrm{~mm}^{2}$. O maior volume de material expelido na soldagem utilizando a condição A obstrui o canal de resfriamento da ferramenta impossibilitando sua função (Figura 5-a). Esse material perdido pode também se alocar entre as partes da ferramenta (Figura 5-b), o que aumenta o torque necessário para rotacionar a camisa e o pino aumentando a energia consumida no processo. Parte desse material expelido pode também ser impulsionado à superfície da amostra depreciando a qualidade superficial da solda (Figura 5-c). 

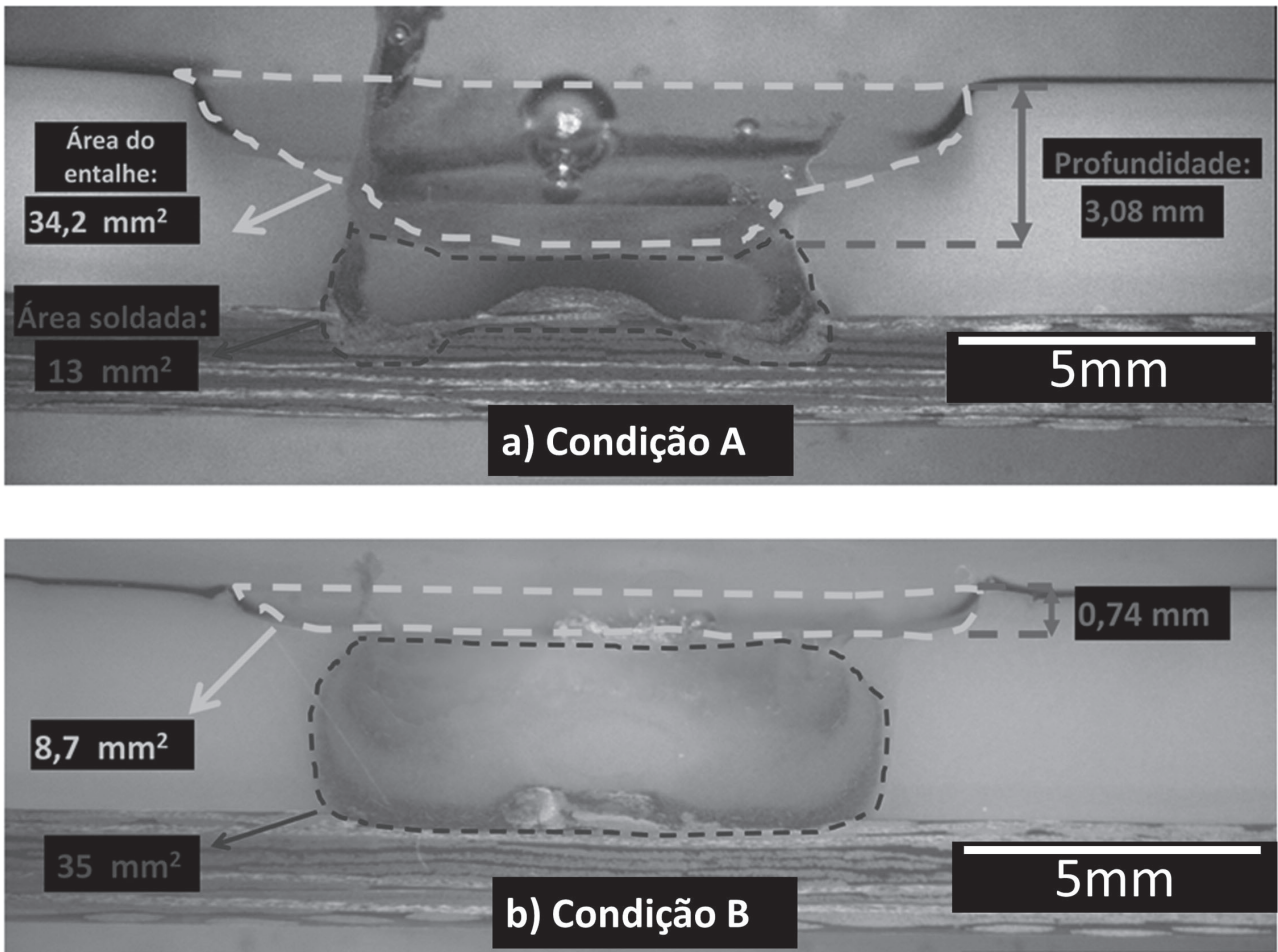

Figura 4. Microestrutura na secção transversal central da solda nas juntas PA6/PA66-CF produzidas por FSpW na condição A (a) e na condição B (b)
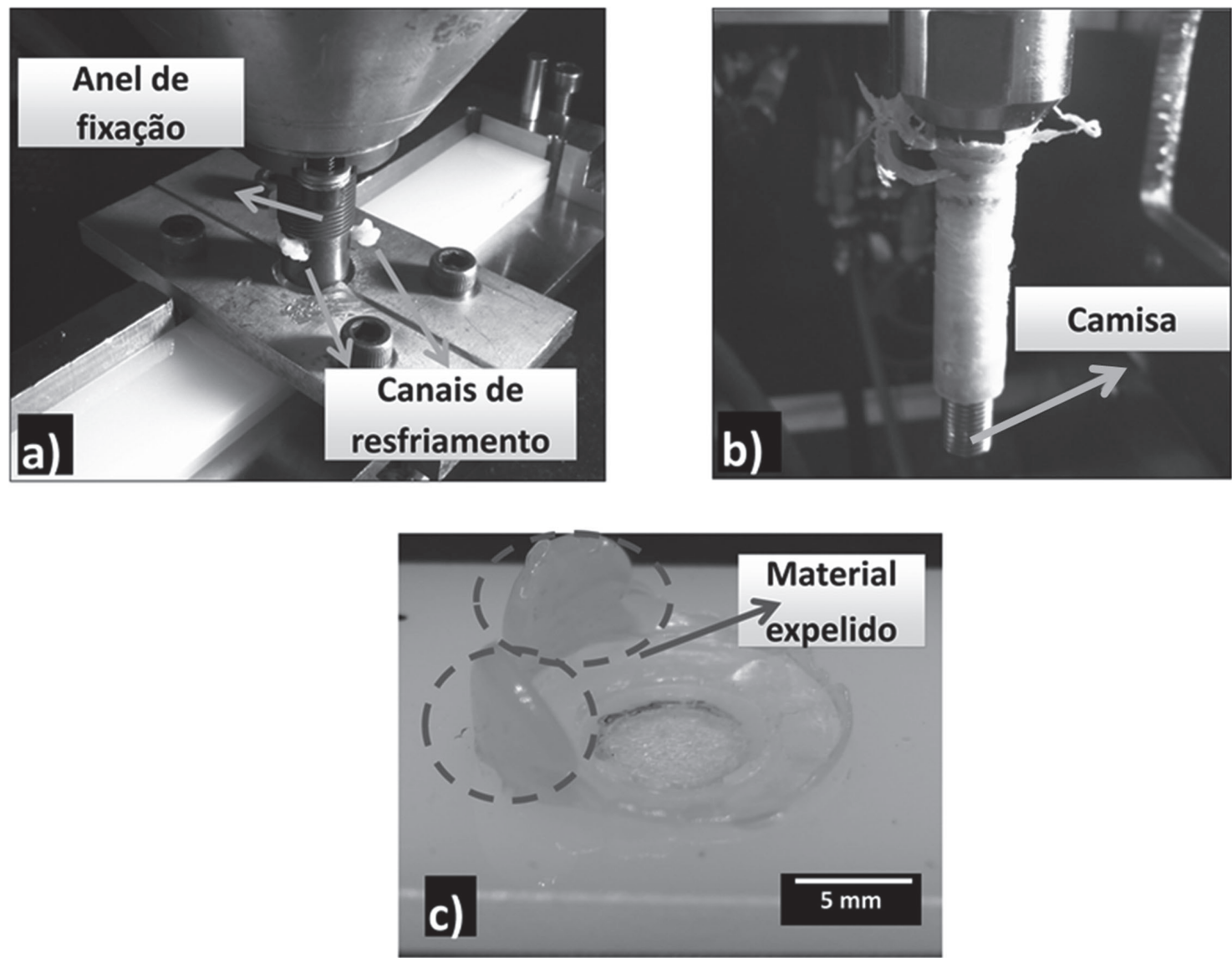

Figura 5. Imagens ilustrando o destino do material descartado durante o processo de soldagem: a) Deposição nos canais de refrigeração; b) Deposição entre o anel de fixação e a camisa; c) Material expelido para a superfície da amostra depreciando o acabamento superficial da solda 
A Figura 6 apresenta os resultados do ensaio de cisalhamento por tração (força máxima e tensão ao cisalhamento) das juntas PA6/CF-PA66 produzidas por FSpW nas condições A e B. A junta produzida na condição B (anel de fixação com diâmetro maior) apresentou resistência ao cisalhamento cerca de $66 \%$ maior (35 $\mathrm{MPa})$ que a junta produzida na condição A (21 $\mathrm{MPa}$ ). O melhor desempenho mecânico da solda produzida na condição B se deve à ausência do entalhe produzindo, assim, menor efeito concentrador de tensão para iniciar trincas, e à maior energia fornecida pelo processo de soldagem devido ao maior tempo de soldagem empregado nessa condição (Tabela 3). A soldagem de termoplásticos por FSpW necessita de maiores aportes térmicos durante a soldagem com relação aos metais,

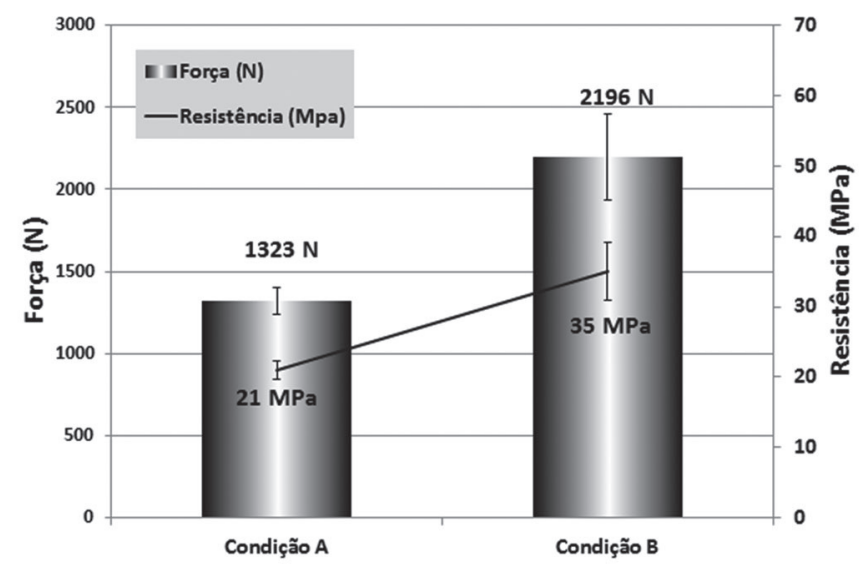

Figura 6. Força máxima e limite de resistência ao cisalhamento das juntas PA6/CF-PA66 produzidas por FSpW nas condições A e B devido à baixa condutividade térmica dos polímeros. Se o maior aporte térmico da condição B fosse utilizado com a geometria da ferramenta otimizada para soldagem em metais, a ferramenta perfuraria toda a amostra impossibilitando a soldagem. Portanto, a modificação proposta na nova dimensão da ferramenta serviu também para aumentar os limites dos parâmetros do processo de soldagem. Maiores tempos de soldagem levam a um maior volume de material fundido que permite uma maior área transversal soldada (Figura 4), possibilitando a obtenção de soldas com melhor desempenho mecânico (Figura 6).

A Figura 7 apresenta os tipos de fratura ocorridos após ensaio de resistência ao cisalhamento das juntas PA6/CF-PA66 produzidas por FSpW, nas condições A (Figura 7a) e B (Figura $7 b-c)$. No caso da solda produzida na condição A, utilizando o anel de fixação com menor diâmetro, verificou-se uma menor espessura de polímero na região da solda o que levou à menor resistência mecânica da solda. Nessa fratura (Fig. 7a), o centro superior da solda se destaca da placa superior de PA6 através da nucleação de uma trinca anular na interface entre o volume de material consolidado e não fundido durante o processamento (efeito geométrico similar ao observado em linhas de solda em peças plásticas injetadas). Quando a nova ferramenta (anel de fixação com maior diâmetro) é utilizada (condição B) verifica-se uma maior espessura de polímero na região da solda. A fratura, nesse caso, ocorre geralmente por quebra da placa superior de PA6 (Fig. 7b), resultando numa solda com maior resistência mecânica. Esse tipo de fratura foi reportada por Oliveira et al. [17] em soldas de PMMA com alta resistência mecânica. Os autores observaram que a trinca nucleia na região da zona de mistura (zona microestrutural aonde o polímero é fundido e misturado) e
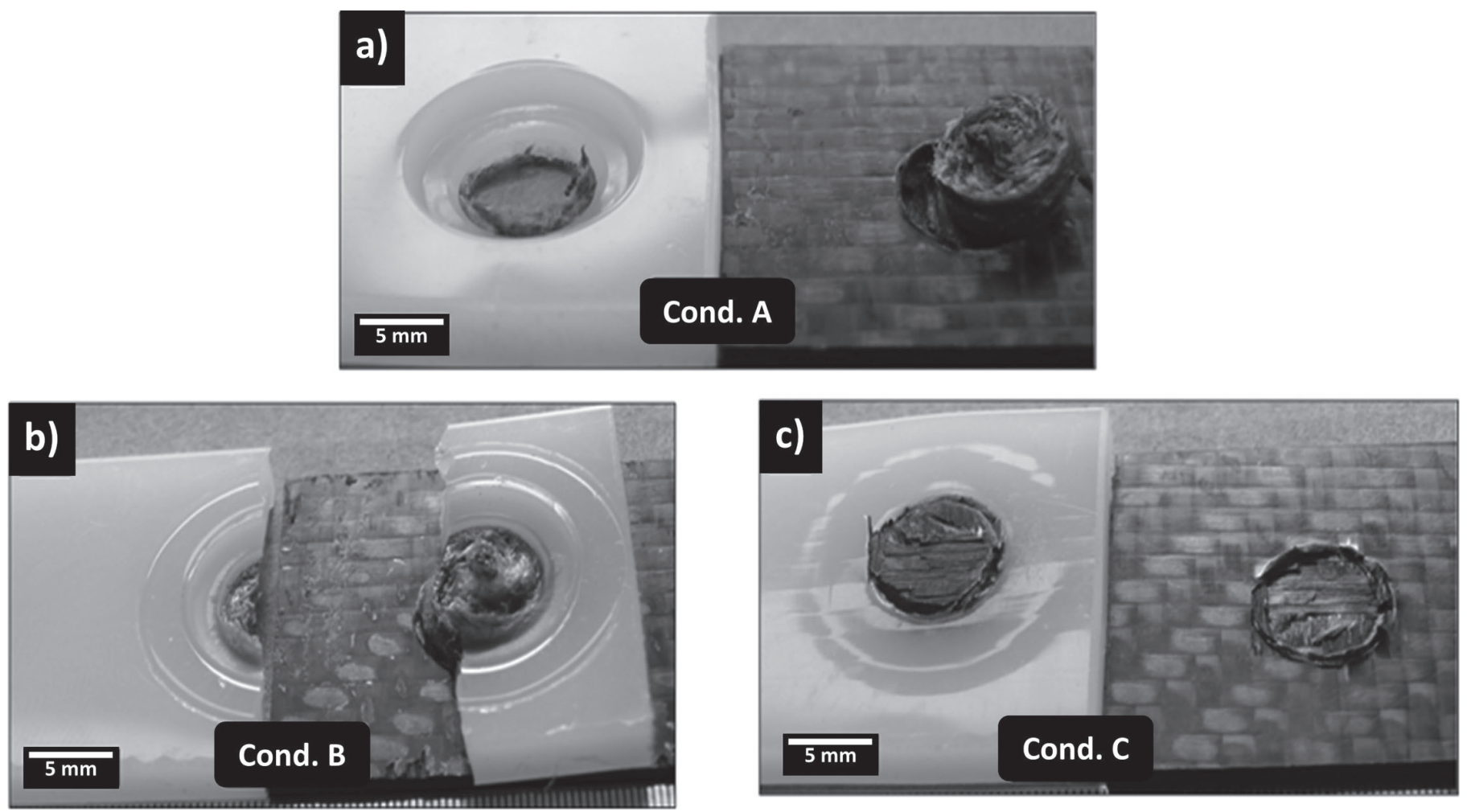

Figura 7. Tipos de fraturas ocorridos após o ensaio mecânico de cisalhamento das juntas PA6/CF-PA66 produzidas por FSpW nas condições A (a) e B (b e c) 
a zona termicamente afetada (aonde o polímero sofre influência térmica, mas as temperaturas processuais permanecem abaixo da transição vítrea do polímero), propagando para o material de base. Outro tipo de fratura observada para a solda PA6/CFPA66 obtida por FSpW na condição B foi por cisalhamento (Fig. 7c) com a trinca nucleando no perímetro da área soldada e propagando em direção ao centro da solda, paralelamente à interface entre as placas superior (PA6) e inferior (CF-PA66). Isso indica uma forte interação da região soldada entre a chapa de PA6 e o laminado CF-PA66, originada da boa mistura entre os materiais e que se deve à otimização dos parâmetros de soldagem. Outro fator que contribuiu para a forte interação da região soldada foi a ausência de defeitos volumétricos e a impregnação de parte das mantas de fibras de carbono na chapa superior de PA6 (Fig. 7c).

\section{Conclusões}

Este trabalho demonstrou pela primeira vez a viabilidade da soldagem de polímeros com compósitos poliméricos pela técnica de soldagem pontual por fricção ('Friction Spot Welding - FSp $W^{\prime}$ ). Foram produzidas juntas PA6/CF-PA66 a partir de condições operacionais otimizadas. $\mathrm{O}$ diâmetro do anel de fixação da ferramenta original (projetada para soldagem de metais) foi modificado visando otimizar o desempenho mecânico e o acabamento superficial das soldas. A utilização da uma nova ferramenta (com diâmetro do anel de fixação maior) possibilitou a seleção de parâmetros de soldagem que resultaram em maior aporte térmico (quantidade de calor) fornecido às amostras durante o processo de soldagem e menor entalhe (inscrição) deixado pela ferramenta na amostra. Esse incremento de energia aumentou o volume de material fundido na área soldada, formando uma solda mais resistente. $\mathrm{O}$ menor entalhe deixado pela nova ferramenta melhorou o acabamento superficial da solda e produziu menor efeito concentrador de tensão para iniciação de trinca, contribuindo também para o melhor desempenho mecânico da solda. O uso da nova ferramenta também permitiu maior precisão na penetração da ferramenta nas chapas sobrepostas e menor desgaste da ferramenta. Verificou-se também que a perda de material expelido no processo diminui, reduzindo a quantidade de material que se aloca entre as ferramentas. Com isso, o sistema de refrigeração do equipamento não é obstruído, a ferramenta sofre menor desgaste por abrasão, o torque para rotação desse material entre as ferramentas diminui e o acabamento superficial da solda não é afetado pelo possível escape de material fundido durante o processo. Com a modificação da ferramenta foi possível estender o intervalo de parâmetros de soldagem de modo que as juntas PA6/CF-PA66 apresentaram um aumento aproximado de 66\% no limite de resistência ao cisalhamento quando comparado com juntas produzidas com a ferramenta padrão, atingindo $35 \mathrm{MPa}$ (2196 N). Como os resultados obtidos foram positivos o projeto demostra a possibilidade de estudos mais aprofundados nessa área, principalmente na investigação de ferramentas com outros diâmetros. Espera-se assim melhorar o desempenho do processo e acelerar a transferência tecnológica da FSpW para a soldagem de polímeros e compósitos poliméricos.

\section{Agradecimentos}

Os autores gostariam de agradecer a Helmholtz Association na Alemanha pelo suporte financeiro (Young Investigator Group "Advanced Polymer-Metal Hybrid Structures") a essa pesquisa e à Coordenação de Aperfeiçoamento de Pessoal de Nível Superior (CAPES) pela bolsa de mestrado do Joel Gonçalves.

\section{Referências Bibliográficas}

[1] AMANCIO-FILHO S.T et al., Recent advances in joining of polymer and polymer-metal hybrid structures by friction-based spot welding techniques. Proceedings of ANTEC 2010, Maio; Orlando, USA, p.1502-1508, 2010.

[2] GIANNOULI. G et al., Impact of European emission control strategies on urban and local air quality. Atmospheric environment, Atmos. Environ; V.45: p.4753-4762, 2011.

[3] SCHILling C., SANTOS J.F., Method and Device for Linking at Least Two Adjoining Work Pieces by Frictional Welding. International Patent WO/2001/036144, Maio, 2005.

[4] AMANCIO-FILHO, S.T et al., Preliminary Investigation of the Microstructure and Mechanical Behaviour of 2024 Aluminium Alloy Friction Spot Welds. Materials Transaction, V.52, p.985-991, 2011.

[5] CAMPANELLI, L.C et al., Parameters optimization for friction spot welding of AZ31 magnesium alloy by Taguchi method/ Otimização dos parâmetros de soldagem por fricção por ponto da liga de magnésio AZ31 pelo método de Taguchi. Soldagem e Inspeção, V.17, p.26-31, 2012.

[6] AMANCIO-FILHO, S.T et al., On the feasibility of friction spot joining in magnesium/fiber-reinforced polymer composite hybrid structures. Materials Science and Engineering, V.528, p.3841-3848, 2011.

[7] ESTEVES J.V et al., Friction spot joining of aluminium 6181-T4 and carbon fiber reinforced poly(phenylene sulfide), Proceedings of ANTEC 2012, Abril 2-4; Orlando, FL, USA, 2012.

[8] GERLICH A; SU P; NORTH T.H. Peak Temperatures and Microstructures in $\mathrm{Al}$ and $\mathrm{Mg}$ Alloy Friction Stir Spot Welds, Sci. and Tech. of Welding and Joining, V.10, No. 6. p.647-652, 2005.

[9] ARICI A; MERT S; Friction Stir Spot Welding of Polypropylene. Journal of Reinforced Plastics and Composites, vol. 00, p.1-4, Maio, 2008.

[10] MITLIN D et al., Structure-properties Relations in Spot Friction Welded (Also Known as Friction Stir Spot Welded) 6111 Aluminum, Materials Science and Engineering, A 441: p.79-96, 2006.

[11] FENG. Z et al., Friction Stir Spot Welding of Advanced High-Strength Steels - A Feasibility Study, SAE Technical Papers, Document No: 2005-01-1248, USA, 2005.

[12] BILICI M.K., Effect of tool geometry on friction stir spot welding of polypropylene sheets. eXPRESS Polymer Letters, V.6, No.10, p.802-813, 2012.

[13] BILICI, M.K., YÜKLER, A., Influence of tool geometry and process parameters on macrostructure and static strength in friction stir spot welded polyethylene sheets, Materials and 
Design, V.33, p.145-152, Janeiro, 2012.

[14] BILICI, M.K., YÜKLER, A., KURTULMUS, M., The Optimization of welding parameters for friction stir spot welding of high density polyethylene sheets, Materials and Design. V.32, Issue 7, p.4074-4079, Agosto (2011).

[15] SILVA A.M., et al., Performance Evaluation of 2-mm thick alclad AA2024 T3 Aluminium Alloy Friction Spot Welding. Buletinul de Informare Documentara (BID-ISIM), V.3, p.36-44, 2007.

[16] OLIVEIRA P.H. et al., Preliminary study on the feasibility of friction spot welding in PMMA. Materials Letter; V.64: p.2098-2101, 2010.

[17] OLIVEIRA, P.H.F et al., Estudo de viabilidade da soldagem de termoplásticos por "Friction Spot Welding" (FSpW). Soldagem e Inspeção, V.17,p.93-103, 2012.

[18] PA6 Erlaton 6, Folha de dados de propriedades técnicas da empresa Arthur Krüger, Alemanha, 2011.

[19] TEPEX Dynalite 201, Folha de dados de propriedades técnicas da empresa BOND Laminates, Alemanha, 2011.

[20] ASTM D3163 - 01(2008) Standard Test Method for Determining Strength of Adhesively Bonded Rigid Plastic LapShear Joints in Shear by Tension Loading, 2008. 